\title{
Optionen für eine «evolutionäre Weiterentwicklung»
}

\section{Klaus-Dieter Hänsgen}

Prof. Dr. sc. nat., Direktor des Zentrums für Testentwicklung und Diagnostik am Psychologischen Institut der Universität Freiburg/Schweiz

Werden die richtigen Personen zum Medizinstudium zugelassen? Universitäten, Kantone und neu der Bund müssen ihre Auswahlziele einbringen, wen sie als Studierende wollen. Diese müssen aber an den Möglichkeiten und Grenzen verschiedener Zulassungsmethoden orientiert bleiben, um weiter Wissenschaftlichkeit gepaart mit Ökonomie bei der Zulassung zu gewährleisten. Ein Überblick möglicher Zulassungsverfahren, ihrer Vor- und Nachteile.

\section{Rahmenbedingungen für NC und EMS in der Schweiz}

Es gibt keinen Grund, an bisherigen Vorteilen oder der Testgüte des Eignungstests für das Medizinstudium (EMS) als kognitivem Studierfähigkeitstests zu zweifeln. Die Absolventenrate liegt an allen Universitäten mit

Options en vue d'un «développement évolutionnaire» Le débat autour des critères d'admission aux études de médecine est tout à fait pertinent. En effet, peuton affirmer que les personnes admises sont les bonnes personnes? Ce débat doit avant tout avoir lieu selon une approche descendante (top-down). A savoir que c'est aux différents acteurs concernés (universités/cantons et désormais aussi la Confédération) d'indiquer les objectifs qu'ils entendent fixer pour choisir les étudiants de demain. Dans un même temps, ils doivent tenir compte des possibilités et des limites des différentes méthodes d'admission en procédant selon une approche ascendante (bottomup) afin de continuer à garantir les critères scientifiques et économiques lors de l'admission. Dans ce contexte, deux questions principales se posent:

- Est-il nécessaire d'adapter les méthodes d'admission utilisées en raison d'un changement général des objectifs de sélection?

- Faut-il diversifier davantage les critères ou appliquer les mêmes critères dans toutes les universités en raison de la multiplicité des objectifs?
Le présent article décrit plusieurs possibilités d'admission ainsi que leurs limites et possibilités de combinaison. Une fois les objectifs fixés, il est possible d'optimiser la procédure.
Numerus clausus (NC) mittlerweile bei über 90\% der Anfänger, die Vorhersagegüte für Studienerfolg ist weiterhin gut, das für die Ausbildung investierte Geld gut angelegt und nicht durch Studienabbrüche verloren. Das Problem war die Testsicherheit (auf eine Aufgabenwiederholung zumindest bis zum befriedigenden $\mathrm{Ab}$ schluss der rechtlichen Auseinandersetzungen muss man nun ganz verzichten); dies wird man also beheben. 2015 wären in allen drei Disziplinen in den vier Universitäten mit NC 2144 Personen zusätzlich zuzulassen gewesen, wenn der NC nicht realisierbar gewesen wäre. Mehrkosten für Medizin würden dafür auf der Basis der IUV-Beiträge (25700 Franken pro Person für das 1. Jahr) minimal in Höhe von 55100800 Franken entstehen. Muss man diese Personen dann mangels Kapazitäten vor allem im Masterstudium wie in der Westschweiz herausprüfen, wären diese Mittel verloren. Wir gehen nachfolgend davon aus, dass Lösungen ohne NC kurzund mittelfristig unrealistisch sind, trotz des geplanten Ausbaus der Kapazitäten bzw. auch bei optimistischem Ausbau. Die Nachfrage nach einem Medizinstudium steigt international an.

Die Rechtsgrundlage des NC ist für Optimierungen des Zulassungsverfahrens ausreichend flexibel:

- In den massgeblichen kantonalen Gesetzgebungen ist die Berücksichtigung der «Eignung» gefordert. Eignungskriterien selbst sind nicht definiert - auch nicht, inwieweit Studien- und/oder Berufseignung zu berücksichtigen ist.

- Die Bildungsziele des Medizinalberufegesetzes MedBG (Kapitel 3) können auch Ziele eines Zulassungsverfahrens definieren, gehen über eine reine Studieneignung hinaus. 
- Die Bundesgerichtsurteile zum NC schreiben zwei weitere wichtige Kriterien fest: «Rechtsgleichheit und Willkürfreiheit».

Jeder bedeutende Eignungstest dieser Welt verfügt über eine Trainingsszene, dies lässt sich kaum verhindern. Auch jeder andere Test oder jede andere Zulassungsmethode hätte diese bald wieder. Für den EMS gilt eine bedingte Trainierbarkeit. Zahlreiche andere Testkonzepte (Wissensprüfung, auch Sozialverhalten oder Interviews) wären wesentlich besser trainierbar. Die Existenz einer Trainingsszene spricht daher nicht gegen die Anwendung von Tests.

\section{Basismodelle für die Zulassung aufgrund von Kriterien}

Welche Varianten sind für eine Zulassung mit NC möglich, wo sind die "Stellschrauben", um die richtigen Personen zuzulassen? Wenn (wie in den meisten Ländern) ein NC und damit eine Selektion notwendig sind, kann man folgende Grundmodelle für die Verwendung von Zulassungskriterien unterscheiden:

- Ein einziges Kriterium für die Zulassung; dieses kann homogener (z.B. kognitive Studierfähigkeit; Schweiz) oder heterogener (Kombination aus kognitiven Leistungen, Schulwissen und sozialem Wissen; Österreich) zusammengesetzt sein.

- Mehrere Kriterien für die Zulassung, meist verbunden mit festen prozentualen Zulassungsquoten

- Parallel bzw. unabhängig voneinander, man hat in mehreren Quoten eine Chance (z.B. Deutschland: Abiturdurchschnitt, Test, Wartezeit), ggf. auch Kombinationen wie Abiturnote plus Test;

- Sequentiell bzw. abhängig mit der Möglichkeit der Vorselektion z.B. durch einen Test und nachfolgender Anwendung aufwendiger Methoden nur für einen eingeschränkteren Personenkreis (z.B. Israel: zuerst ein Test, dann ergebnisabhängig Einladung eines Teils zu Interviews).

Die Verwendung eines homogenen einzelnen Kriteriums birgt die Gefahr der Einseitigkeit der Auswahl die «Typenvielfalt» der Personen, die wegen unterschiedlicher Interessen unterschiedliche Berufsanforderungen bedienen können, wird möglicherweise eingeschränkt. Dies vermutet man in der Schweiz (sollte auch empirisch untersucht werden, da bisher Belege fehlen):

- Aktuell werden nur noch die (auf Studienanmeldung bezogen) 25\% der Leistungsbesten in Humanmedizin an den NC-Universitäten zugelassen - aufgrund der seit 2001 kontinuierlich steigenden Studiennachfrage wird der Anteil zukünftig ggf. noch geringer.
- Leistungsbeste definieren sich aus den Faktoren des EMS, in denen kognitive Fähigkeiten der Studieneignung dominieren, "schlussfolgendes Denken" der zentrale Faktor neben Gedächtnis, Aufmerksamkeit und visuellen Fähigkeiten ist.

- Die Prognosekraft des Tests für das Bestehen der Prüfungen in der Regelstudienzeit ist für die Leistungsbesten am höchsten. Aufgrund der «Verschärfung» des NC werden nun auch Personen nicht zugelassen, die von den kognitiven Leistungen her ebenfalls gute Aussichten hätten, das Studium erfolgreich abzuschliessen. Deren Stärken können ggf. auf anderen Gebieten liegen, welche die "Merkmalsdiversität» unter den Studierenden bereichern würden.

- Dies vermutet man vor allem für Personen, die sozial bzw. vom Engagement oder der Einsatzbereitschaft her geeigneter sind und unterstellt meist, dass sehr gute Personen beim "schlussfolgernden Denken» weniger sozial geeignet seien (das Klischee der «Intelligenzbestie» steht irgendwo dahinter). Soziale Intelligenz/Kompetenz, Engagement, Motivation oder andere "Soft-Skills» und gute kognitive Leistungen schliessen sich aber nicht aus, korrelieren stattdessen sogar positiv. Will man zur Erhöhung der Merkmalsdiversität bei den Studierenden auch Beste nach diesen Kriterien erfassen, muss man diese explizit berücksichtigen und vorher mit ausreichender Güte erheben können.

Bei der bisherigen Diskussion zur Erweiterung der Kriterien gibt es zwei auf den ersten Blick gegensätzliche Richtungen, die mit den politischen Zielen der Zulassung abgeglichen werden müssen. Spätestens hier stellt sich auch die Frage nach der Qualität der Ausbildung und dem Stellenwert einer soliden mathematisch-naturwissenschaftlichen Grundausbildung.

- Mehr Hard-Skills: Verwendung von Wissensfragen (Mathematik, Physik, Chemie, Biologie). Statt der Fähigkeit zur Wissensaneignung (quasi der «Beschleunigungsfähigkeit») würde der Wissensstand selbst (quasi die aktuelle "Geschwindigkeit») erfasst. Es gibt Klagen an allen Universitäten, dass das Ausgangsniveau sehr heterogen sei und das "niedrigste» Niveau als Einstieg für die Lehrveranstaltungen gewählt werden muss, was Probleme bei der Stoffvermittlung insgesamt verursacht. Naturwissenschaftskenntnisse aus der Matura sind nicht zwingend für eine Bewerbung zum Medizinstudium - etwa, wenn man einen anderen Schwerpunkt gewählt hat. Medizin bleibt aber ein diesbezüglich anspruchsvolles Studium - nicht nur für das Prüfungsbestehen, sondern auch die spätere Berufsausübung. Eine «Anforderungsreduktion» (etwa für Hausärzte oder Psychiater) auf diesem Gebiet wird folglich von vielen 
Fachleuten abgelehnt, um keine «zweitklassigen» Mediziner auszubilden.

- Vorteile wären, dass entweder durch entsprechende frühe Schwerpunktwahl in der Matura bzw. durch das zusätzliche «Training» für den Test ein effektiver Nutzen für das Studium entstünde und die beklagte Heterogenität im Wissensstand ausgeglichen würde. Eine frühe Orientierung auf die Studien- und Berufsanforderungen wird folglich nicht als Problem oder Einschränkung der Wahlfreiheit bei der Matura oder deren Abwertung gesehen. Zum Beispiel können Interessenten für ein Sportstudium auch nicht bis zum Bewerbungszeitpunkt warten, ehe sie mit einem intensiven Training beginnen. Hat man diesen Schwerpunkt nicht gewählt, könnte und müsste man dies rechtzeitig nacharbeiten. «Training» auf diesem Gebiet hätte einen Nutzen.

- Ein Nachteil kann sein, dass sich "extensiv» angeeignete Vorkenntnisse (Kurse bzw. Training, frühere Berufsausbildungen oder Studiengänge) eine Studieneignung vorspiegeln, die nicht vorhanden ist, und man im realen Medizinstudium dann quasi nicht so gut mitkommt und nicht besteht.

- Mehr Soft-Skills: Es gibt vor allem von ausserhalb der Medizinausbildung die Wünsche, "Soft-Skills» stärker zu berücksichtigen. Soziale Kompetenz, Engagement und Einsatzbereitschaft sowie andere Merkmale, die man von einem "guten Arzt» erwartet, gehören dazu. Dadurch will man vor allem die Berufseignung stärker berücksichtigen. Es gibt dazu aber sehr wenige konkrete Anforderungsanalysen; die Merkmale wirken teilweise wie Idealisierungen, mit denen man sich den aufopferungsvollen guten alten Hausarzt zurückwünscht - aber bestimmte gesellschaftliche Veränderungen übersieht. Medizinalberufe sind in sich schon sehr heterogen. Dennoch wäre es denkbar, dass zumindest ein Teil der Kandidaten über bessere Voraussetzungen bezüglich Motivation und Interesse verfügt. Diese würde man nur durch einen Einbezug in das Zulassungsverfahren finden.

Methoden zur Erfassung von Soft-Skills in Selektionssituationen, die auf Selbstbeurteilung beruhen, sind anfällig für Antworten nach der sozialen Erwünschtheit, was zugleich eine gut trainierbare Funktion ist. Dies misst dann eher Lippenbekenntnisse oder die schauspielerischen Fähigkeiten. Wir bieten aktuell SelfAssessments an, wo man sich anonym Klarheit über die Passfähigkeit Persönlichkeit/Interessen/Wissen über Studium und Beruf verschaffen kann - Ehrlichkeit ist eine Voraussetzung für ein verwertbares Ergebnis. Dies wird sehr gut genutzt, hat aber keinen unmittelbaren Einfluss auf die Zulassung und wäre für die
Fragebogenteile so auch nicht in einem selektiven Test verwendbar [1]

- Situational Judgement Tests (SJT) (Vorgabe einer Situation und dazu Multiple-Choice-Verhaltensalternativen zur Auswahl) werden in einigen Ländern eingesetzt, auch die Association of American Medical Colleges (zuständig für den MCAT-Test) in den USA evaluiert diese und gibt einen Überblick, wo dieser Ansatz verwendet wird. Wir hatten auf eine geplante Pilotstudie gemeinsam mit einem der führenden Entwickler verzichtet, weil die Probleme überwogen: Entweder wird schon berufsspezifisches Wissen vorausgesetzt, es dominiert die soziale Erwünschtheit der richtigen Antworten oder der Konsens ist zu wenig eindeutig und kulturabhängig, was «richtiges» Verhalten ist. Hier werden wir den aktuellen Stand weiter analysieren, ob es neue verwendbare Entwicklungen gibt. Beispiele, wo sich auch die Probleme «soziale Erwünschtheit» bzw. «Voraussetzung berufsspezifischer Kenntnisse» zeigen, findet man in einem österreichischen Übungsportal.

- Interviews ohne oder mit standardisierten Aufgabenstellungen (z.B. Vorgabe einer Situation, auf die im Gespräch reagiert werden muss - man nennt dies Assessments) bleiben eine zweite Möglichkeit, die vielerorts angewendet wird. Ein Beispiel aus Deutschland ist der HAM-INT. Die Probleme des optimalen Verhaltens in den dort vorgegebenen Situationen sind dem SJT vergleichbar, wobei das Verhalten schwerer "verstellt» werden kann als beim schriftlichen Beantworten von Fragen. Aus Belgien ist bekannt, dass die Präsentation der Aufgaben in Videoform besser war als die schriftliche. Das richtige Erkennen nichtverbaler Informationen kann eine Rolle spielen - wie es in Interviews und Assessments auch notwendig ist.

Hier nehmen Interviewer die Beurteilungen aufgrund ihrer Beobachtung vor, was Schulung bzw. Training und ausreichende Beurteilerübereinstimmung erfordert. Diese Methoden sind aufwendig und werden ohne Vorselektion nicht bezahlbar sein (in Deutschland geschieht dies z.B. mittels des Schulwissenstests HAM-NAT, auch in Israel funktioniert dies mit einem Test). Sie müssten an den Universitäten durch qualifiziertes akademisches Personal durchgeführt werden. Dabei wären ggf. auch unterschiedliche Vorgehensweisen und Quoten für die einzelnen Universitäten und damit unterschiedliche Schwerpunktbildungen möglich.

Bekannt ist aus einer Metaanalyse, dass die Prognosekraft für Studienerfolg im Vergleich zu Test und Maturitätsnoten deutlich geringer ist. Die Begründung für einen Einbezug müsste sein, dass Merk- 


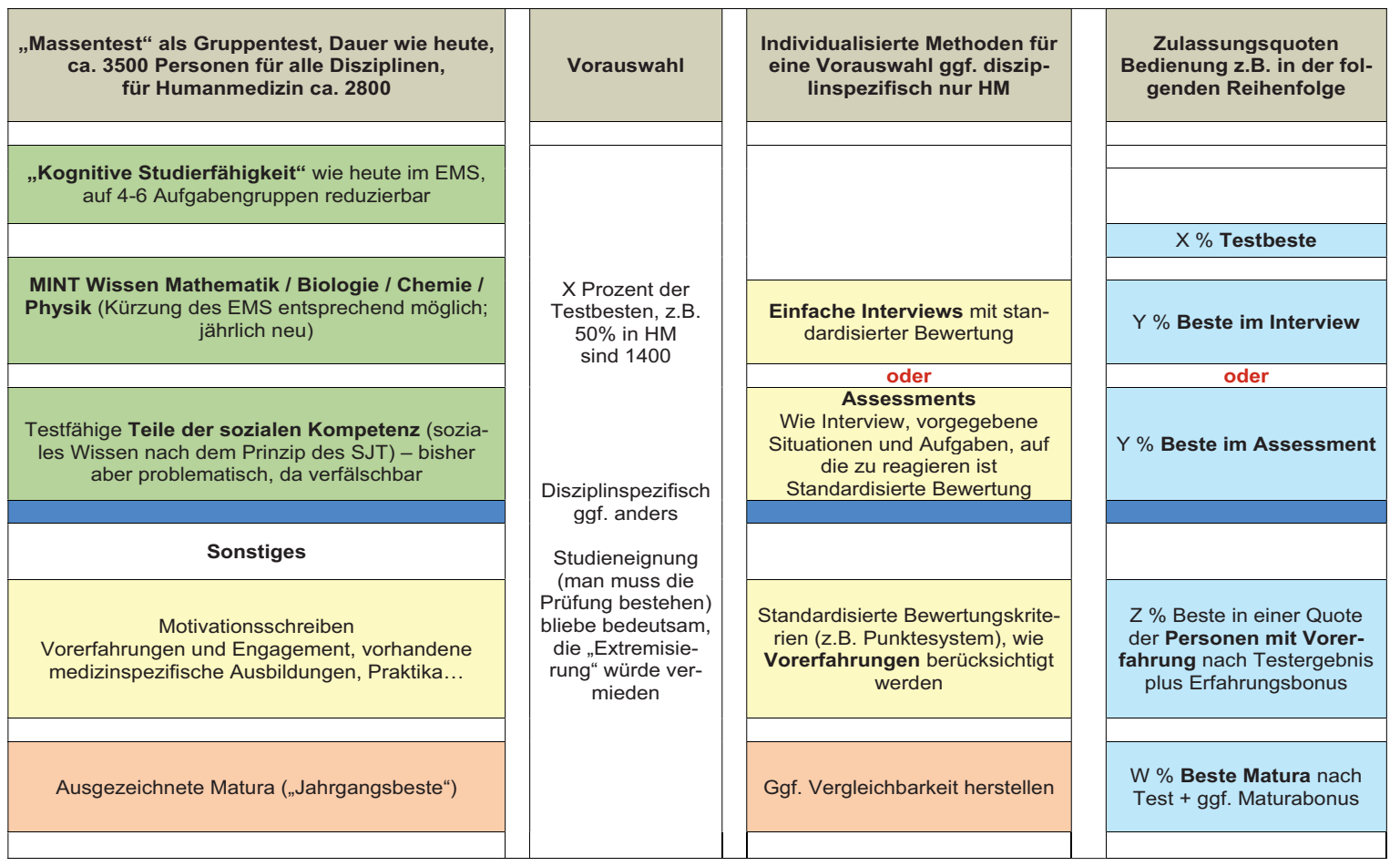

Tabelle 1: Synopse mehrerer international üblicher Verfahren der Zulassung zum Medizinstudium.

male gemessen werden, die heute den Studienerfolg in den ersten Jahren weniger beeinflussen - die Bedeutung, die sich aus der Perspektive der Berufseignung ausreichend begründen lässt.

Begrenzend wirkt die Ökonomie: Zwei geschulte Beurteiler und 30 Minuten pro Person scheinen ein Minimum, um nicht nur an der Oberfläche zu «kratzen». Ein Team kann nicht mehr als 16 Personen pro Tag beurteilen. Lädt man 300 Personen ein (rund $10 \%)$, sind rund 20 «Teamtage» notwendig.

- Berücksichtigung des Engagements wäre über sogenannte Motivationsschreiben möglich. Verifizierbare berufliche Vorerfahrung, Praktika, gesellschaftliches Engagement im Hinblick auf Medizinalberufe u.Ä. sowie die Begründung des Berufswunsches werden ebenfalls von Beurteilern bewertet und können in einer eigenen Quote zur Zulassung führen - wieder nach dem Testergebnis oder Bonifizierung des Testergebnisses. Hier wären die klare Definition für die Gewährleistung von Rechtsgleichheit und Willkürfreiheit sowie eine realistische Einschätzung der Verfügbarkeit (z.B. chancengleiche Praktikumsstellen für alle - was aufgrund der grossen Personenzahl problematisch sein kann) wichtig. Maturanoten sind nach wie vor problematisch, weil sie zwischen Kantonen und Schultypen nicht vergleichbar sind und Rückwirkungen auf das Benotungssystem befürchtet werden («Kantönligeist»). Dennoch gibt es auch Beschwerden, dass Jahrgangsbeste dann beim Test nicht die gleiche Leistung zeigen und nicht zugelassen werden, obwohl hinter der Maturanote «jahrelange erfolgreiche Anstrengung» steht. Allenfalls wäre es denkbar, über eine feste und anfangs geringe Quote diejenigen Personen zuzulassen, die eine besonders exzellente Prüfung (z.B. Jahrgangsbeste) abgelegt haben - dies ebenfalls nach dem EMS-Ergebnis. Die Vergleichbarkeit von Exzellenz, ihr Nachweis und Rückwirkungen auf die "Auszeichnungspraxis» wären dabei zu bedenken.

\section{Wie könnte man das Zulassungs- kriterium weiterentwickeln?}

NC und der EMS werden im Auftrag der Kantone resp. Universitäten als Dienstleistung durchgeführt. Diese sind die eigentlichen "Stakeholder». Letztendlich müssen diese vorgeben, welche Studierende sie wollen, die zum Ausbildungsprofil passen. Es muss ein Gremium gefunden werden, wo Stakeholder und «Zulassungsexperten" sich in einer Art moderiertem Prozess auf eine Lösung einigen, wie die Zulassungsziele machbar und sowohl wissenschaftlich als auch ökonomisch vertretbar sind sowie rechtsfähig umgesetzt werden. Strukturen für diese Diskussion wären zu schaffen. Die Tabelle 1 enthält als Synopse mehrerer international üblicher Verfahren die Optionen, deren Prüfung wir aufgrund des aktuellen Diskussionsstandes vorschlagen würden. Sie ist quasi die «Speisekarte», Basis für die Auswahl der Kriterien durch die «Stakeholder».

1 samed.unifr.ch (allgemein) sowie www.sazamed.ch (Zahnmedizin der Universität Basel). 